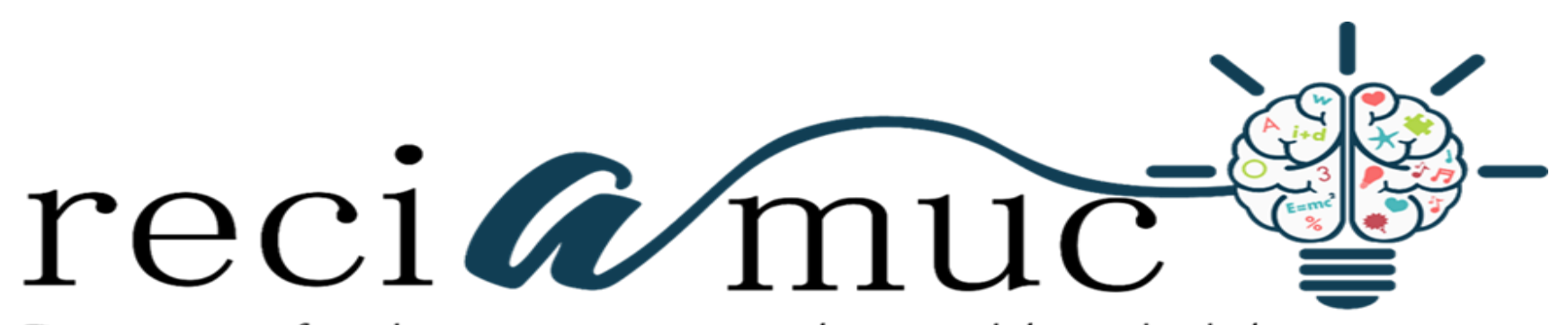

Revista científica de investigación actualización del mundo de las ciencias

Melissa Paulina Pereira Pontón a ; Jheison David Cabrera Ruilova ${ }^{\text {b }}$; María José

Miranda Laborda ${ }^{c}$; Lenin Eduardo Granda Álvarez ${ }^{\mathrm{d}}$

Efectos de la vitamina D en prevención del asma

Effects of vitamin $D$ in prevention of asthma

Revista Científica de Investigación actualización del mundo de las Ciencias. Vol. 3

núm., 2, abril, ISSN: 2588-0748, 2018, pp. 781-810

DOI: $10.26820 / \mathrm{reciamuc/3.(2).abril.2019.781-810}$

URL: http://reciamuc.com/index.php/RECIAMUC/article/view/366

Código UNESCO: 3206.13 Vitaminas

Tipo de Investigación: Artículo de Revisión

(C) RECIAMUC; Editorial Saberes del Conocimiento, 2019

Recibido: $15 / 01 / 2019$

Aceptado: 07/02/2019

Publicado: 01/04/2019

Correspondencia: melpereira88@ hotmail.com
a. Médico; Investigador Independiente; Guayaquil, Ecuador; melpereira_88@hotmail.com
b. Médico; Investigador Independiente; Guayaquil, Ecuador; jheisoncabrera.r@gmail.com
c. Médico; Investigador Independiente; Guayaquil, Ecuador; mj.Mirandalaborda@ @otmail.com
d. Médico; Investigador Independiente; Guayaquil, Ecuador; slashyhero@ gmail.com 


\section{Efectos de la vitamina $D$ en prevención del asma}

Vol. 3, núm. 2., (2019)

Melissa Paulina Pereira Pontón; Jheison David Cabrera Ruilova; María José Miranda Laborda; Lenin Eduardo Granda Álvarez

\section{RESUMEN}

Todas las funciones que el cuerpo humano realiza para cumplir sus actividades diarias son importantes, es así como, la respiración, que permite obtener oxígeno para el cuerpo y expulsar el dióxido de carbono. Pero, debido al estrechamiento de las vías respiratorias surge el asma, enfermedad caracterizada por que los receptores que revisten a los bronquios perciben sustancias específicas que contraen o relejan los músculos subyacentes y producen alteración del flujo de aire. La misma es una respuesta ante la sensibilidad anómala de los receptores colinérgicos que desencadenan una contracción en los músculos de las vías respiratorias cuando no deben hacerlo. En otras palabras, los mastocitos ubicados en los bronquios son responsables del inicio de dicha respuesta al expulsar sustancias como la histamina y leuctrienos que aumentan la secreción de moco, desplazan ciertos glóbulos blancos hacia la zona en cuestión. Asimismo, los eosinófilos presente en los pacientes asmáticos liberan sustancias adicionales que incrementan el estrechamiento de la vía respiratoria, ante estos eventos, los especialistas recomienda el uso de la vitamina $\mathrm{D}$, que ofrece cierta protección contra las crisis asmáticas, reduce la exacerbación de los músculos lisos de los bronquios que causan su estrechamiento; para así, ayudar a los pacientes a realizar un menor esfuerzo para respirar; pues, las contracciones musculares de las vías respiratorias se detienen, desaparece la inflamación y estas vías respiratorias se ensanchan de nuevo, permitiendo el flujo de aire hacia dentro y hacia fuera de los pulmones y recuperan la normalidad. Planteamientos que apoyan el desarrollo del presente artículo ubicado en una investigación documental, apoyada en fuentes determinadas por la lectura y revisión de informaciones encontradas en documentos tecnológicos y materiales impresos, que permitieron alcanzar su propósito de analizar los efectos de la vitamina D en prevención del asma, para luego elaborar las conclusiones del mismo.

Palabras claves: Vitamina D; Prevención asma; Vía respiratoria. 


\title{
Efectos de la vitamina $D$ en prevención del asma
}

Vol. 3, núm. 2., (2019)

Melissa Paulina Pereira Pontón; Jheison David Cabrera Ruilova; María José Miranda Laborda;

Lenin Eduardo Granda Álvarez

\begin{abstract}
All the functions that the human body performs to accomplish their daily activities are important, so, as the breathing, which allows getting oxygen to the body and expelling carbon dioxide. But, due to narrowing of the Airways is the asthma, a disease characterized by that the receptors that line the bronchi perceive specific substances that contract or relaxing the underlying muscles and cause alteration of the flow of air. It is a response to the abnormal sensitivity of cholinergic receptors that trigger a contraction in the muscles of the Airways when they should do so. In other words, the mast cells located in the bronchi are responsible for the onset of the response to expel substances such as histamine and leuctrienos, which increases the secretion of mucus, moving certain white blood cells to the area in question. Also, eosinophil in asthmatic patients released additional substances that increase the narrowing of the airway, before these events, the specialists recommend the use of vitamin $\mathrm{D}$, which offers some protection against the asthma, reduces the exacerbation of the smooth muscles of the bronchi causing narrowing; so, help patients make less effort to breathe; so stop the muscle contractions of respiratory, inflammation disappears and these Airways are widened again, allowing the flow of air into and out of the lungs and recover normal. Approaches that support the development of this article located in a documentary research, supported by sources determined by reading and revision of information found in technological documents and printed materials, which allowed achieve their purpose of analyzing the efe.
\end{abstract}

Key Words: Vitamin D; Preventing asthma; Respiratory tract. 


\section{Efectos de la vitamina $D$ en prevención del asma}

Vol. 3, núm. 2., (2019)

Melissa Paulina Pereira Pontón; Jheison David Cabrera Ruilova; María José Miranda Laborda; Lenin Eduardo Granda Álvarez

\section{Introducción.}

El asma es una enfermedad causada por una inflamación crónica del tracto respiratorio. Durante la misma, el cuerpo se encuentra sometido a estrés oxidativo. Esto conduce a pensar que la ingesta de altas cantidades de vitaminas antioxidantes, a través del consumo de frutas y verduras o de suplementos alimenticios tendrá un efecto favorable en el curso de esta enfermedad. Asimismo, se puede decir que es un problema mundial de salud pública, se han estimado en el mundo 334 millones de personas que sufren de asma, contrario a lo establecido esta patología tiene cifras de prevalencia altas en países industrializados, en la actualidad son los habitantes de países en desarrollo los más afectados, donde también la incidencia crece de manera notable Su incremento está directamente relacionado con cambios en la dieta, como menor ingesta de frutas y verduras frescas, aunado al incremento en el consumo de harinas refinadas, carnes rojas y grasas saturadas.

Cabe destacar que, la respuesta inflamatoria en la vía aérea es activada debido a la presencia de estímulos alérgenos, como: la temperatura ambiental, tabaco, bacterias o virus, infecciones respiratorias o estímulos mecánicos como el ejercicio. Muchas de las células involucradas en este proceso (eosinófilos, neutrófilos, monocitos y macrófagos) generan especies reactivas de oxígeno (ERO), como los superóxidos (O2 -). Estos al ser eliminados por la enzima superóxido dismutasa generan peróxido de hidrógeno (H2O2). Ambas especies químicas forman radicales libres potentes en los sistemas biológicos al reaccionar con biomoléculas, causando daño celular. Por ello, las especies reactivas de oxígeno (ERO) son compuestos altamente oxidantes que combaten al agente 


\section{Efectos de la vitamina $D$ en prevención del asma}

Vol. 3, núm. 2., (2019)

Melissa Paulina Pereira Pontón; Jheison David Cabrera Ruilova; María José Miranda Laborda; Lenin Eduardo Granda Álvarez

agresor. Además, la concentración de óxido nitroso en las vías aéreas del paciente asmático se encuentra incrementada.

Es importante decir, que el esputo de personas con asma suele estar presente una cantidad mayor de especies reactivas de oxígeno (ERO) y especies reactivas de nitrógeno (ERN) que en aquel proveniente de personas sanas. Ambas, causan daño tisular al reaccionar con proteínas, ADN y lípidos del epitelio respiratorio: pueden inducir apoptosis, alterar la proliferación celular e interferir con funciones del sistema inmune en los pulmones y provocan broncoconstricción e incremento del moco. Además de los antioxidantes endógenos mencionados, el paciente asmático está sometido a radicales libres provenientes del ambiente.

En consecuencia, para contrarrestar el daño oxidativo, en el pulmón se desarrolla una defensa antioxidante que neutraliza los radicales libres, evitando el daño celular. Estas moléculas pueden ser enzimas como superóxido dismutasa, catalasa y glutatión-peroxidasa o compuestos no enzimáticos como el glutatión, vitaminas $\mathrm{E}$ y $\mathrm{C}$ y carotenos, principalmente. Muchos fluidos provenientes del pulmón contienen numerosos antioxidantes; estos también pueden clasificarse como endógenos (glutatión, enzimas antioxidantes) o exógenos (vitaminas A, C y E). Cuando las especies oxidantes superan a las antioxidantes se rompe la homeostasis redox y esto genera un estado conocido como estrés oxidativo que es determinante en la severidad del asma.

Es importante destacar que, el organismo adquiere la vitamina D de algunos alimentos como pescado (salmón y atún), queso, mantequilla y yema de huevo. Sin embargo, la fuente más importante de vitamina $\mathrm{D}$ proviene de la producción endógena en la piel a partir del 7 dihidrocolesterol. Este precursor, gracias a la acción de la luz ultravioleta, se convierte en 


\section{Efectos de la vitamina $D$ en prevención del asma}

Vol. 3, núm. 2., (2019)

Melissa Paulina Pereira Pontón; Jheison David Cabrera Ruilova; María José Miranda Laborda; Lenin Eduardo Granda Álvarez

colecalciferol o vitamina D1. A nivel hepático, la enzima 25 hidroxilasa agrega un grupo hidroxilo al colecalciferol transformándolo en 25 hidroxicolecalciferol, que es la forma de depósito de la vitamina $\mathrm{D}$ en el hígado y tejido adiposo. Posteriormente la $25(\mathrm{OH})$ vitamina $\mathrm{D}$ llega al riñón donde actúa la 1 alfa hidroxilasa que le agrega un segundo grupo hidroxilo, dando origen finalmente a la forma activa de la vitamina D, la 1, 25 dihidroxicolecalciferol.

Al tomar en consideración los planteamientos anteriores, se puede indicar que como resultados a los cambios en los estilos de vida del ser humano y el bajo consumo de alimentos que proveen al organismo de la Vitamina $\mathrm{D}$, se han incrementado la presencia de las enfermedades respiratorias como el asma, dado que, en los últimos 40 años se ha observado una disminución progresiva de los niveles de vitamina D en la población, los cuales se encuentran asociado a aumento del asma bronquial. Tal como lo asevera Soto (2018) precisa que se han observado:

Niveles bajos de vitamina D y se correlacionan con un aumento de la hiperreactividad bronquial y atopia. En niños con diagnóstico establecido de asma bronquial, bajos niveles de vitamina D se asocian a evolución severa, incluye mayor número de consultas a Servicio de Urgencia y hospitalizaciones, baja función pulmonar, mayor uso de corticoides inhalados y sistémicos y exacerbaciones más frecuentes y severas. (p.22)

Según lo indicado, la acción de la vitamina $\mathrm{D}$ en el asma bronquial estaría dada por su papel inmunomodulatorio sobre las células del sistema inmune, inhibiendo citokinas del tipo Th2, inhibiendo la secreción de IL 11 y estimulando la de IL10. Además la vitamina D tendría cierto papel en revertir la corticoresistencia y remodelación de la vía aérea. Cabe agregar, que la principal funcion de la vitamina D en el cuerpo humano, es la esquelética, pues, la misma provee el balance 


\section{Efectos de la vitamina $D$ en prevención del asma}

Vol. 3, núm. 2., (2019)

Melissa Paulina Pereira Pontón; Jheison David Cabrera Ruilova; María José Miranda Laborda; Lenin Eduardo Granda Álvarez

de calcio y fósforo necesario para mantener una adecuada función ósea. Por ello, cuando se presentan bajos niveles se muestra una disminución del epitelio respiratorio, haciendo posible el surgimiento del asma bronquial.

Asimismo, Alonso (2017) destaca que “desde el punto de vista epidemiológico se ha observado un aumento del número de personas que tienen niveles sanguíneos insuficientes y deficientes de vitamina D y al mismo tiempo un aumento progresivo de la prevalencia de asma" (p.8) Estas ideas, llevan a destacar que el incremento del asma bronquial, tiene una respuesta epidemiológico, determinada por la carencia de la vitamina D, es decir, dicha insuficiente involucra a una mayor cantidad de pacientes con la enfermedad.

De igual manera, Alaei (2019) precisó en su estudio que:

Los pacientes asmáticos la enfermedad fue de 13,1\% de los niños hospitalizados. Su gravedad estuvo representada en un $43,4 \%$. El 16,1\% de los niños menores de cinco años se clasificaron como de alto riesgo y no controlada en el 47,7\%. Los niños asmáticos tuvieron niveles séricos de vitamina D significativamente inferior en comparación con los controles $(14.53 \pm 8.10$ $\mathrm{ng} / \mathrm{ml}$ frente a $22.45 \pm 13.46 \mathrm{ng} / \mathrm{ml} ; \mathrm{p}<0.001)$. La prevalencia de deficiencia de vitamina $\mathrm{D}$ en el grupo de asma fue de $73.6 \%$ y $49.1 \%$ en los controles; mientras que las cifras respectivas de suficiencia de vitamina D fueron de $7.5 \%$ y del $32.1 \%$ e insuficiencia de vitamina D fueron similares $(18.9 \%) \cdot(\mathrm{p} .15)$

Al considerar los datos arrojados por el autor, se puede deducir que si bien la presencia de asma se asoció significativamente con niveles séricos disminuidos de vitamina D, no hubo una 


\section{Efectos de la vitamina $D$ en prevención del asma}

Vol. 3, núm. 2., (2019)

Melissa Paulina Pereira Pontón; Jheison David Cabrera Ruilova; María José Miranda Laborda; Lenin Eduardo Granda Álvarez

asociación entre la gravedad del asma o la falta de control de la enfermedad con la deficiencia de vitamina D. En los menores de 5 años no hubo diferencias entre los subgrupos de bajo y alto riesgo. El riesgo de asma en los niños con deficiencia de vitamina D fue 6.3 veces más que en aquellos con niveles normales de vitamina D. Por ello, se puede considerar que, es posible que el uso de suplementos con vitamina D ayude a la deficiencia de la misma y puede evitar la progresión del asma. Al respecto, Alaei (ob.cit) indica que "la vitamina D cumple funciones importantes en la regulación de las respuestas inmunológicas involucradas en la inflamación asmática”. (p.18).

En esta misma dirección el último autor citado, precisa que es importante considerar que los problemas vinculados con la sobre ingesta de vitamina D pueden acarrear, el incremento en la producción de catelicidina, un péptido antimicrobiano. Un incremento sustancial en esta producción puede tener un efecto proinflamatorio y ocasionar cambios en la microbiota, lo que deriva en un efecto negativo para el asma, puesto que la microbiota es una barrera de defensa contra patógenos en todo el epitelio, incluyendo el respiratorio. De allí, la necesidad que cuya medicación, debe ser cumplida bajo vigilancia médica, con el fin de evitar problemas asociados con aquellas enfermedades relacionadas con las vías respiratorias.

Cada uno de los aspectos expuestos en párrafos anteriores, sirven de aporte para el desarrollo del presente artículo, mediante el cual se busca llegar a un análisis preciso y coherente en cuanto a los efectos de la vitamina D en la prevención del asma, argumentos que permiten valorar el significado médico de estimar su utilización mediante suplementos encargados de ofrecer beneficios para el fortalecimiento del sistema inmune y garantizar con ello la reducción de 


\section{Efectos de la vitamina $D$ en prevención del asma}

Vol. 3, núm. 2., (2019)

Melissa Paulina Pereira Pontón; Jheison David Cabrera Ruilova; María José Miranda Laborda; Lenin Eduardo Granda Álvarez

aquellos procesos inflamatorios de las vías respiratorias ante la presencia de estímulos alergénicos, a fin de ofrecer a los pacientes mejores niveles de calidad de vida.

\section{Método.}

La sistematización de los diferentes argumentos en el desarrollo científico, se encuentran determinados por la presencia de un método encargado de orientar su recorrido científico en relación a un tema en particular; en este caso, al buscar analizar los efectos de la vitamina D en la prevención del asma, se considera pertinente la aplicación del concepto dado por Valdez (2019) representa "el campo o sendero que a manera de una construcción teórica guía al investigador a conseguir determinados objetivos en su tiempo preciso con actividades que tienen ventajas de disciplinar la acción humana.”(p.54).

Por lo tanto, la selección del método no es más que una respuesta escogida para aprender la realidad con todas sus connotaciones y por lo tanto, depende de la creatividad del investigador para utilizar de manera exitosa los mejores procedimientos que le faciliten interpretaciones objetivas de ese contexto previamente seleccionado, es decir, hacer posible una modificación en cuanto a la evaluación de los contenidos que se necesitan para organizar los aspectos que estructuran el desarrollo integral del artículo. En este caso particular, el investigador precisa lo planteado por Valdez (ob.cit) en relación al método crítico "hace posible redimensionar las apreciaciones de otros investigadores para convertirlos en nuevos esquemas que facilitan la construcción del desarrollo del problema al poder concluir o generalizar”. (p.57) 


\section{Efectos de la vitamina $D$ en prevención del asma}

Vol. 3, núm. 2., (2019)

Melissa Paulina Pereira Pontón; Jheison David Cabrera Ruilova; María José Miranda Laborda; Lenin Eduardo Granda Álvarez

En respuesta a las consideraciones dadas en el contexto del método e incorporación como herramienta básica en todo proceso científico, lleva entonces a ubicar el proceso investigativo en un campo especial del hacer interpretativo. Es decir, se fijan posiciones claras en cuanto al tipo, técnicas documentales y técnicas para la recolección de información que hacen posible el desarrollo del tema seleccionado previamente.

\section{Tipo de Investigación.}

El conocimiento científico, puede ser entendido en dos direcciones, la primera como el acto de comprender una realidad que está caracterizada por diferentes elementos propios que al ser valorados logran darle consistencia investigativa y la segunda como un proceso final que hace posible fijar posiciones claras ante los acontecimientos debidamente interpretados, para así configurar sus estructuras metodológicas. En otras palabras, estas acciones llevan al investigador hacia la organización de sus interpretaciones bajo el marco de una tipo de investigación especial.

En consecuencia, el contenido temático fijado para la construcción de este artículo, estima la correspondencia con la investigación documental definida por Arias (2012)

Es un proceso basado en la búsqueda, recuperación, análisis, crítica e interpretación de datos secundarios, es decir, los obtenidos y registrados por otros investigadores en fuentes documentales: impresas, audiovisuales o electrónicas. Como en toda investigación, el propósito de este diseño es el aporte de nuevos conocimientos. (p.27)

Según lo citado por Arias, se precisa que todo proceso investigativo enmarcado en lo documental, busca una interpretación de diferentes criterios de forma crítica, estimado por la 


\section{Efectos de la vitamina $D$ en prevención del asma}

Vol. 3, núm. 2., (2019)

Melissa Paulina Pereira Pontón; Jheison David Cabrera Ruilova; María José Miranda Laborda; Lenin Eduardo Granda Álvarez

selección de aquellos materiales impresos que dan oportunidad de crear nuevas consideraciones generales en cuanto a un tópico debidamente establecido por el investigador para así elaborar nuevos conocimientos científicos.

De este modo, la incorporación de datos especiales, se convierten en una unidad de información que se obtiene durante la ejecución de una investigación. Según su procedencia, los datos se clasifican en primarios, cuando son obtenidos originalmente por el investigador; y secundarios, si son extraídos de la obra de otros investigadores, para así llegar a la selección de las técnicas documentales que dan sustentación al desarrollo de los contenidos previamente organizados para finalmente llegar a formular las respectivas conclusiones vinculadas con el tema previsto en su desarrollo.

\section{Fuentes Documentales.}

El contenido temático que ha sido previamente seleccionado, no sólo fija las condiciones metodológicas del artículo, sino que encamina las respectivas ubicaciones de sus aspectos generales; es así como, una vez relacionado su método y tipo de investigación, se comprende la significación que presentan las técnicas documentales, mediante las cuales, el investigador lograr darle la respectiva ubicación a cada uno de los contenidos preestablecidos y así finalmente diseñar el respectivo soporte del material a presentar.

En esta dirección, Arias (ob.cit) define las técnicas documentales como "todas aquellas acciones que suministran información, pueden ser aportadas por documentos, obras, material tecnológico, libros donde se registran informaciones de interés para construir un tema en especial". 


\section{Efectos de la vitamina $D$ en prevención del asma}

Vol. 3, núm. 2., (2019)

Melissa Paulina Pereira Pontón; Jheison David Cabrera Ruilova; María José Miranda Laborda; Lenin Eduardo Granda Álvarez

(p.32). De allí, que el tema previamente seleccionado para el desarrollo de este artículo, al estar relacionado con los efectos de la vitamina $\mathrm{D}$ en la prevención del asma, lleva al investigar a indagar, revisar, leer, interpretar diferentes documentos referidos al contenido, para luego desglosarlos en apreciaciones generales que dan cabida a nuevas interpretaciones desde una perspectiva innovadora.

\section{Técnicas de Recolección de Información.}

Dentro del campo científico relativo a la investigación documental, las fuentes encargadas de darles consistencia teórica al estudio de un tema en particular, se encuentran determinadas por la introducción de técnicas que le permiten al investigador proyectar sus apreciaciones hacia otros horizontes. Es así como, Arias (ob.cit) las considera como "medios o materiales, impresas, audiovisuales y electrónicas, que permiten la organización de los contenidos informativos que dan relevancia científica a un tema en general”. (p.36)

Al considerar, el último planteamiento, se puede indicar que para el desarrollo del presente artículo, se asumieron como técnicas de recolección de información, la revisión en los diferentes medos electrónicos, así, como la revisión de libros y revistas que sirvieron para complementar cada uno de los aspectos previamente establecidos en función a la necesidad del tema por redactar y de esta manera, crear nuevos criterios científicos de interés para otros investigadores que manifiesten la necesidad de revisión de sus aspectos generales. 


\section{Efectos de la vitamina $D$ en prevención del asma}

Vol. 3, núm. 2., (2019)

Melissa Paulina Pereira Pontón; Jheison David Cabrera Ruilova; María José Miranda Laborda; Lenin Eduardo Granda Álvarez

\section{Resultados.}

La actividad de búsqueda de información relativa al tema previamente seleccionado por el investigador, permite fijar una posición crítica - reflexiva en cuanto al desarrollo del cuerpo temático. Por ello, su organización se hizo de manera particular con el fin de ofrecer al lector diferentes eventos que le ofrezcan al final la oportunidad de comprender su generalización de manera precisa al criterio manejado durante su realización.

\section{Sistema Respiratorio}

La vida de los seres humanos para ser desarrollada de manera efectiva, requiere de la presencia de diferentes funciones básicas que hacen posible la conducción de sus actividades diarias; por ello, para comprender la aparición del asma como enfermedad bronquial, es determinante precisar las características del sistema respiratorio. El cual se encuentra conformado por un conjunto de órganos encargados de cumplir la respiración, así como otras funciones, entre ellos se citan los siguientes:

Fosas Nasales: Son dos cavidades que se encuentran en el interior de la nariz, cuya función es permitir la entrada del aire, el cual se humedece, filtra y calienta a través de unas estructuras llamadas cornetes.

Faringe: Es un tubo musculoso situado en el cuello y revestido de membrana mucosa; conecta la nariz y la boca con la tráquea y el esófago. Por ella, pasan tanto el aire como los alimentos, por lo que forma parte tanto del aparato digestivo como del aparato respiratorio. Mide 


\section{Efectos de la vitamina $D$ en prevención del asma}

Vol. 3, núm. 2., (2019)

Melissa Paulina Pereira Pontón; Jheison David Cabrera Ruilova; María José Miranda Laborda; Lenin Eduardo Granda Álvarez

unos trece centímetros, extendido desde la base externa del cráneo hasta la $6^{\circ}$ o $7^{\circ}$ vértebra cervical, ubicándose delante de la columna vertebral.

Epiglotis: Es la estructura del cuerpo que cuelga hacia abajo de la tráquea. Cuando el alimento se traga, la epiglotis impide que penetren en los pulmones. En ausencia de Epiglotis una persona puede ahogarse y toser cada hora mientras se come.

Laringe: Este órgano permite el paso del aire desde la nariz hacia la tráquea y de ahí a los pulmones. Es una estructura móvil, que forma parte de la vía aérea, actúa normalmente como una válvula que impide el paso de los elementos deglutidos y cuerpos extraños hacia el tracto respiratorio inferior. Además, permite el mecanismo de la fonación para la producción de la voz. La emisión de sonidos está condicionada al movimiento de las cuerdas vocales. Son los movimientos de los cartílagos de la laringe los que permiten variar el grado de apertura entre las cuerdas y una depresión o una elevación de la estructura laríngea, con lo que varía el tono de los sonidos producidos por el paso del aire a través de ellos. Esto junto a la disposición de los otros elementos de la cavidad oral (labios, lengua y boca) permite determinar los diferentes sonidos que emitimos.

Tráquea: Es una parte muy importante del aparato respiratorio, es el tubo que conecta la nariz y la boca con los bronquios y los pulmones. Cuándo una persona inspira (toma aire) el aire entra por la boca o nariz y pasa a la laringe, de aquí pasa a través de la tráquea para llegar a los bronquios y finalmente a los pulmones. Su papel es el de ofrecer una vía abierta al exterior desde los pulmones. Debido a este papel fundamental en la respiración, cualquier daño en la tráquea es potencialmente muy peligroso para la vida. 


\section{Efectos de la vitamina $D$ en prevención del asma}

Vol. 3, núm. 2., (2019)

Melissa Paulina Pereira Pontón; Jheison David Cabrera Ruilova; María José Miranda Laborda; Lenin Eduardo Granda Álvarez

Bronquio: Uno de los dos conductos tubulares en que se bifurca la tráquea y por los que se introduce el aire en los pulmones. Conduce el aire desde la tráquea a los bronquiolos y estos a los alvéolos.

Bronquiolos: Son parte de las vías respiratorias en los pulmones. Están situados en el extremo de los bronquios, la ramificación más grande de las vías respiratorias en los pulmones, y terminan en los alvéolos, pequeños sacos circulares donde el oxígeno se intercambia con dióxido de carbono en la sangre. Estos pasajes se distinguen de los bronquios en que no contienen cartílago o glándulas. Son responsables de controlar la distribución del aire y la resistencia del flujo de aire en los pulmones. El aire es aspirado dentro de los pulmones a través de las vías respiratorias superiores hasta que alcanza los alvéolos, donde oxigena la sangre. La sangre desoxigenada transfiere su dióxido de carbono en los alvéolos, y el dióxido de carbono se respira a través de las vías respiratorias.

Pulmones: Son un par de sacos (derecho e izquierdo) que se encuentran en la cavidad torácica y que llevan a cabo la función de la oxigenación sanguínea. Sirven para separar el Oxigeno de otras sustancias toxicas para luego transportarlo a la sangre. Son los encargados de transformar el aire que respiramos en oxígeno, que será transportado a través del sistema cardiovascular por la sangre a todas las células del organismo. El pulmón derecho es el más grande, ya que el izquierdo tiene que cederle una parte de su espacio para acogerla corazón. Los mismos, se hinchan unos quinientos millones de veces durante una vida para atraer aire fresco (oxígeno O2) y expulsar aire usado (dióxido de carbono o CO2). Están protegidos por la caja torácica y se apoyan sobre el diafragma. 


\section{Efectos de la vitamina $D$ en prevención del asma}

Vol. 3, núm. 2., (2019)

Melissa Paulina Pereira Pontón; Jheison David Cabrera Ruilova; María José Miranda Laborda; Lenin Eduardo Granda Álvarez

Músculos Intercostales: Mueven la caja torácica y permiten la respiración pues al contraerse los músculos flexores provocan la espiración y al contraerse los músculos extensores, la inspiración. Agrupan a las láminas musculares que ocupan los espacios comprendidos entre dos costillas vecinas. Se ubican en la parte anterior y lateral del tórax y su función es actuar en los movimientos respiratorios.

Diafragma: Es un músculo de forma alargada que separa la cavidad torácica de la abdominal; se sitúa debajo de los pulmones en forma de cúpula y su función es, ni más ni menos, intervenir en la respiración. Al inhalar, este poderoso músculo se contrae y se achata aumentando la capacidad torácica y creando un vacío que atrae mayor cantidad de aire a los pulmones, y en la exhalación se relaja y recupera su forma de cúpula a medida que los pulmones expulsan el aire.

Con el fin de representar gráficamente la estructura del sistema respiratorio, se presenta a continuación la figura $\mathrm{n}^{\circ} 1$ que le ofrece al lector una visualización precisa de los diferentes órganos encargados de facilitar las funciones respiratorias en los individuos. 


\section{Efectos de la vitamina $D$ en prevención del asma}

Vol. 3, núm. 2., (2019)

Melissa Paulina Pereira Pontón; Jheison David Cabrera Ruilova; María José Miranda Laborda;

Lenin Eduardo Granda Álvarez

Figura $\mathbf{N}^{\circ} 1$ Sistema Respiratorio

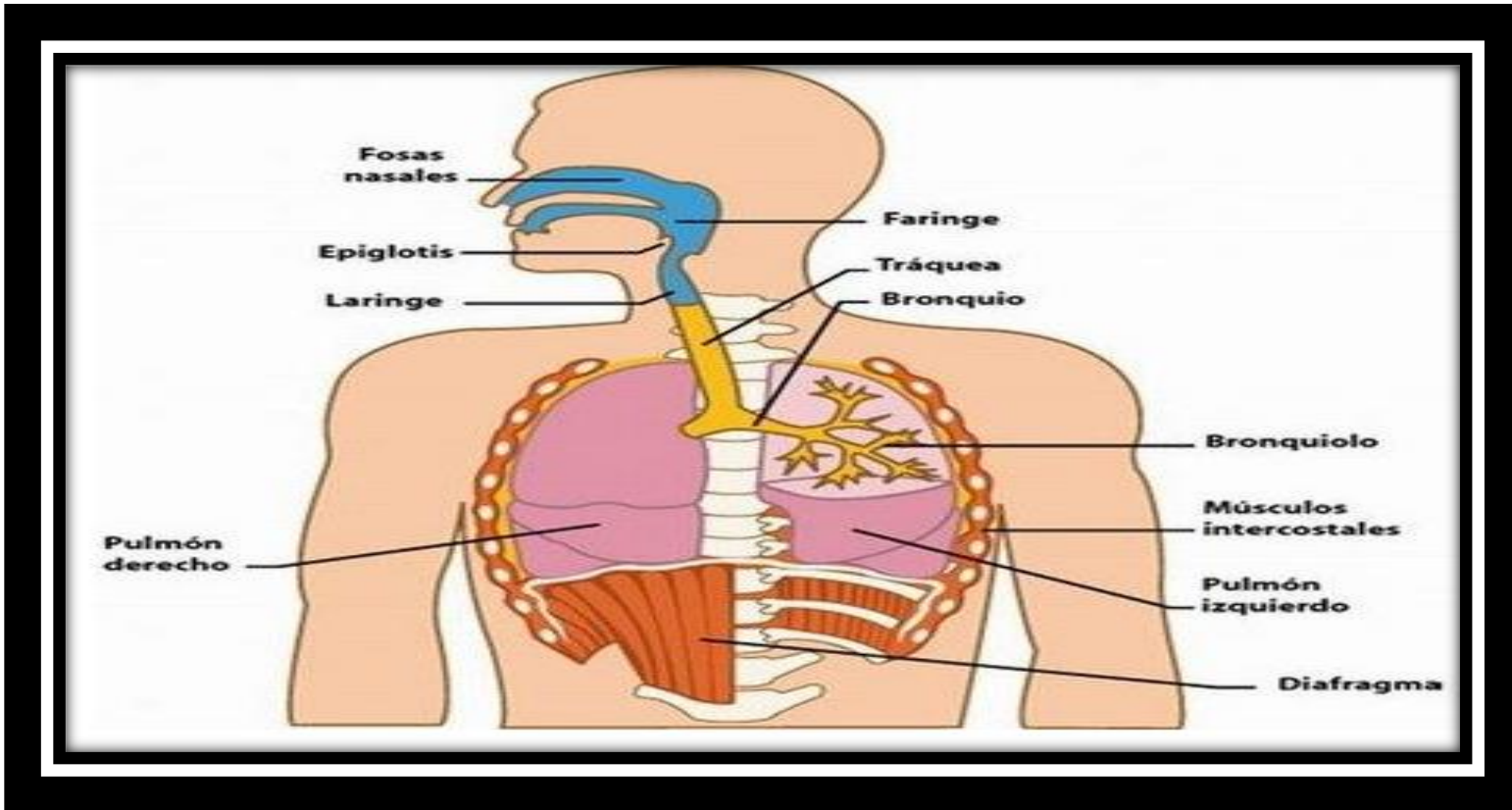

Fuente: Soto (ob.cit)

Figura $\mathbf{N}^{\circ} 2$ Procesos de la Respiración

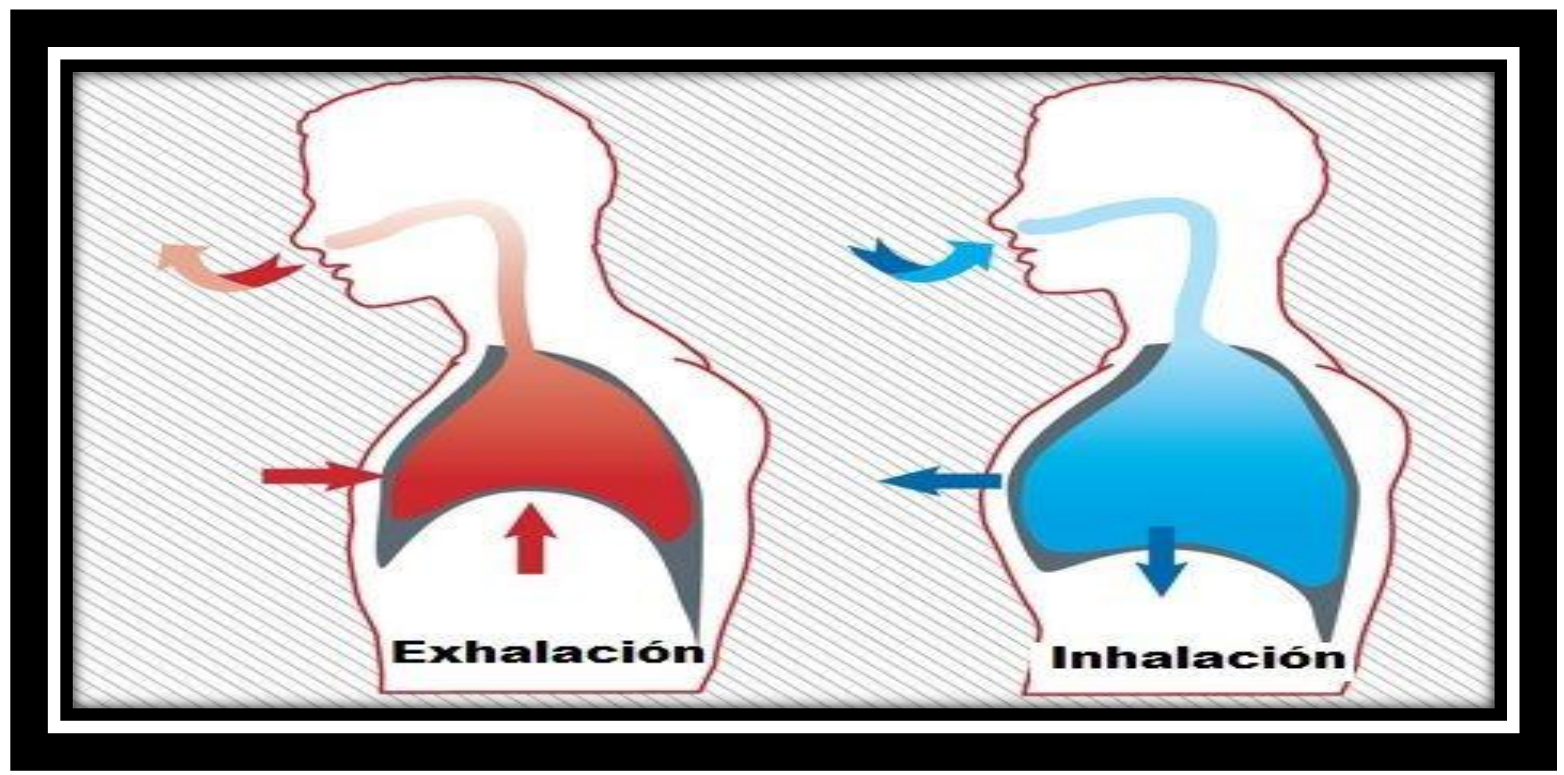

Fuente: Soto (ob.cit) 


\section{Efectos de la vitamina $D$ en prevención del asma}

Vol. 3, núm. 2., (2019)

Melissa Paulina Pereira Pontón; Jheison David Cabrera Ruilova; María José Miranda Laborda; Lenin Eduardo Granda Álvarez

En función a lo que aprecia en la figura $\mathrm{n}^{\circ} 2$, se puede decir que el proceso de respiración para cumplirse de manera idónea, debe realizar la inhalación, que no es más que llevar aire a los pulmones. Tomamos aire para los pulmones. Además, ayuda al cuerpo a llevar el oxígeno y la exhalación ayuda al cuerpo a expulsar el dióxido de carbono. Mientras que la exhalación, empuja el aire fuera de los pulmones.

Por ello, cuando las funciones del sistema respiratorio son afectadas por cualquier estímulo o receptor, aparecen las llamadas enfermedades crónicas, entre las cuales se encuentran: las pulmonares, obstrucción crónica, de origen laboral, hipertensión pulmonar, rinitis alérgicas, y el asma. Según la Organización Mundial de la Salud (2015) "se estiman que 235 millones de personas padecen asma y mueren alrededor de 4 millones de individuos por enfermedades respiratorias crónicas". (p.24). Estas apreciaciones son de interés para el desarrollo del tópico asma que caracteriza al presente artículo

Asma

El asma es un problema de salud pública grave con una prevalencia conservadora a nivel mundial de 235 millones de personas. La carga económica (>18 mil millones de \$ al año en EE. UU.) y humanitaria del asma afecta de forma más intensa a los países en desarrollo, en los que la gestión tiende a ser más deficiente, lo que aumenta el riesgo de exacerbaciones y sus consecuencias, como pueden ser las hospitalizaciones y el absentismo. En los países desarrollados, los miembros más vulnerables de la sociedad, como los niños y las minorías, presentan un mayor riesgo de padecer asma. El asma en Latinoamérica ocupa un $13 \%$ de la superficie terrestre y alberga aproximadamente a 600 millones de personas. 


\section{Efectos de la vitamina $D$ en prevención del asma}

Vol. 3, núm. 2., (2019)

Melissa Paulina Pereira Pontón; Jheison David Cabrera Ruilova; María José Miranda Laborda; Lenin Eduardo Granda Álvarez

A pesar de que se suele contemplar como un bloque cultural homogéneo, la región es muy diversa y cuenta con una gran variedad de características sociodemográficas, diferentes idiomas y religiones, y servicios de salud con estructuras y recursos dispares. Actualmente, el conocimiento que hay en la región acerca de la prevalencia del asma y de los factores que la provocan es precario. Los factores de riesgo podrían ser específicos en cada región; por ejemplo, la contaminación y la dieta en los países de Europa del Este y la obesidad y el estrés en los países de Occidente. La diversidad de Latinoamérica hace que todos los factores de riesgo existentes estén localizados dentro de la región.

La exposición a los factores de riesgo de padecer asma podría originarse en la etapa prenatal por causa del humo del tabaco y dieta materna. Varios de los factores sensibilizadores postnatales incluyen la exposición a ácaros, tabaco, contaminación, ozono, infecciones y carencias de vitamina D. Los datos relativos a Latinoamérica sugieren que factores sociales como la exposición a la violencia, podrían, también, incidir en un aumento de la prevalencia del asma de 8 a 10. Los tres factores principales que predisponen a dicha prevalencia probablemente sean la genética, contaminación y exposición temprana a las infecciones virales. Estos factores de riesgo tan genéricos comparten una consecuencia común: el aumento de los cambios pro-inflamatorios, que conforman la base de la fisiopatología del asma. 


\section{Efectos de la vitamina $D$ en prevención del asma}

Vol. 3, núm. 2., (2019)

Melissa Paulina Pereira Pontón; Jheison David Cabrera Ruilova; María José Miranda Laborda; Lenin Eduardo Granda Álvarez

Figura $\mathbf{N}^{\circ} 3$ Prevalencia del Asma en Latinoamérica

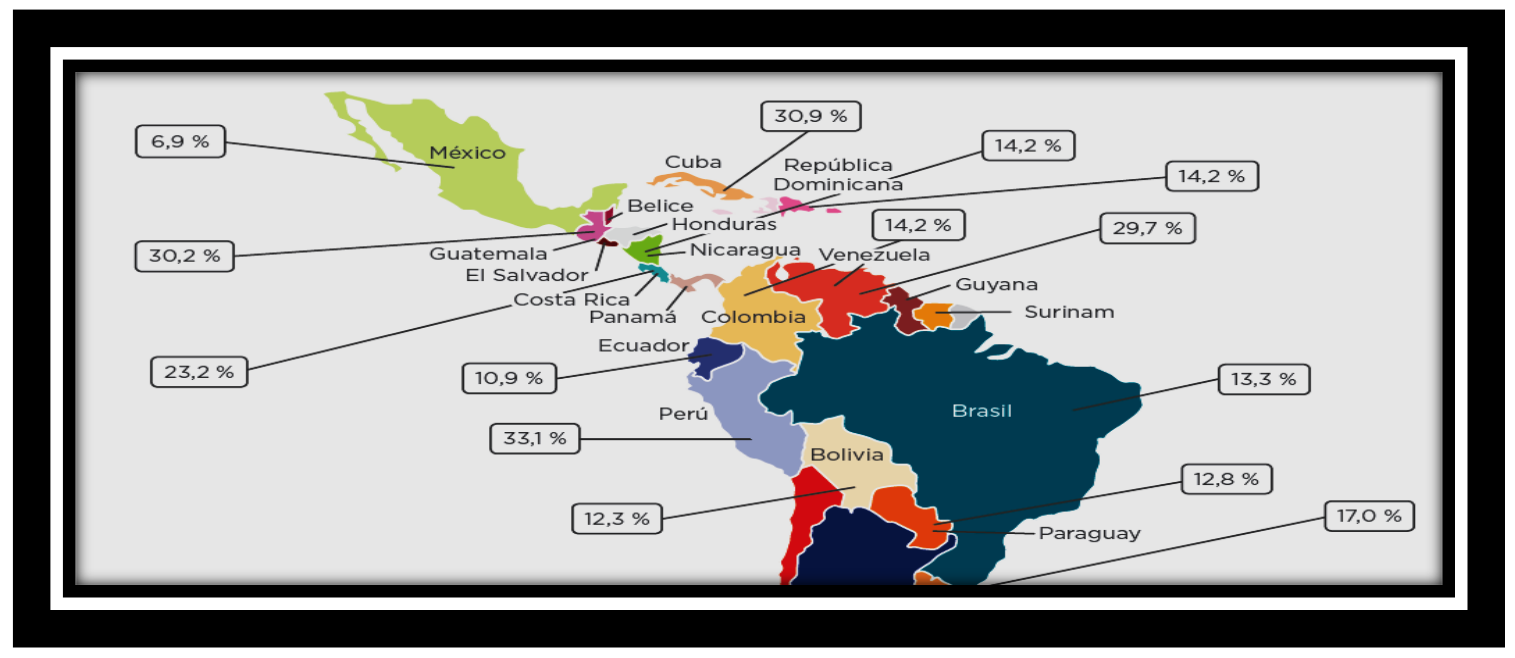

Fuente: OMS (ob.cit)

Cabe destacar que la figura que antecede, lleva a indicar que a pesar de que se suele contemplar como un bloque cultural homogéneo, la región es muy diversa y cuenta con una gran variedad de características sociodemográficas, diferentes idiomas y religiones, y servicios de salud con estructuras y recursos dispares. Actualmente, el conocimiento que hay en la región acerca de la prevalencia del asma y factores que la provocan es precario; sin embargo, los datos que se manejan indican que el asma es un problema de salud significativo en Latinoamérica. De acuerdo con un análisis sistemático reciente, la prevalencia osciló entre el $7 \%$ de México y el $33 \%$ de Perú.

En esta misma dirección, se puede indicar que el asma no está considerada como una enfermedad, sino como un grupo heterogéneo de trastornos que tienen como consecuencia una obstrucción bronquial reversible y recurrente que se caracteriza por la inflamación, la hiperreactividad de las vías respiratorias y la limitación del flujo aéreo. Para la Organización Mundial de la Salud (ob.cit) la definición de "un grupo heterogéneo de trastornos supone todo un 


\section{Efectos de la vitamina $D$ en prevención del asma}

Vol. 3, núm. 2., (2019)

Melissa Paulina Pereira Pontón; Jheison David Cabrera Ruilova; María José Miranda Laborda; Lenin Eduardo Granda Álvarez

reto. La definición clásica del asma caracteriza el trastorno en relación con episodios recurrentes de falta de aire, tos y sibilancias, que tienen lugar, principalmente, por la noche”. (p.18).

Al considerar esta definición, la mayor parte de los pacientes tiene una enfermedad leve, y corre el riesgo de no ser debidamente diagnosticada, de no recibir el tratamiento correcto y carecer de un control adecuado. La definición fisiológica se centra en la limitación del flujo aéreo y en la presencia de biomarcadores inflamatorios. En Latinoamérica, la espirometría para detectar el flujo aéreo está infrautilizada, sobre todo, en niños, y, en algunos casos, ni siquiera está disponible. Finalmente, la definición patológica se centra en la inflamación crónica, con o sin cambios en el flujo aéreo, incluido el remodelado.

Asimismo, la presencia de los microbios es una dicotomía en el riesgo de asma. Para el desarrollo del sistema inmune y para evitar la atopia, es fundamental tener un microbioma equilibrado, mientras que los patógenos infecciosos aumentan el riesgo de desarrollar asma y exacerbaciones. El control del asma implica diferentes medidas para cada tipo de persona. Desde la perspectiva del paciente, el control puede significar vivir una vida plena, poder ir a trabajar o al colegio, participar en actividades y dormir tranquilo. El médico suele centrarse en los resultados a largo plazo, por ejemplo, evitar el uso excesivo de esteroides, debido a las consecuencias que pueden tener, con el tiempo, en la densidad de los huesos. Desde el punto de vista del sistema sanitario en general, la reducción de costes y carga de la enfermedad constituyen la panacea. Por suerte, el mantenimiento del control fisiológico y evitación de exacerbaciones suponen el logro de todos estos objetivos a largo plazo. 


\section{Efectos de la vitamina $D$ en prevención del asma}

Vol. 3, núm. 2., (2019)

Melissa Paulina Pereira Pontón; Jheison David Cabrera Ruilova; María José Miranda Laborda; Lenin Eduardo Granda Álvarez

En este sentido, Paz (2018), define al asma como una enfermedad respiratoria caracterizada por "inflamación crónica de las vías aéreas (bronquios) que causa episodios recurrentes de sensaciones de falta de aire (disnea), pitos en el pecho con la respiración (silibancias), tos y sensación de opresión en el pecho". (p.32). Es decir, sus manifestaciones se encuentran determinada por una obstrucción reversible y ocasional de la vía aérea que, si no se trata adecuadamente, puede llevar a una obstrucción permanente.

Figura $\mathbf{N}^{\circ} 4$ Bronquiolos Normales y Asmático

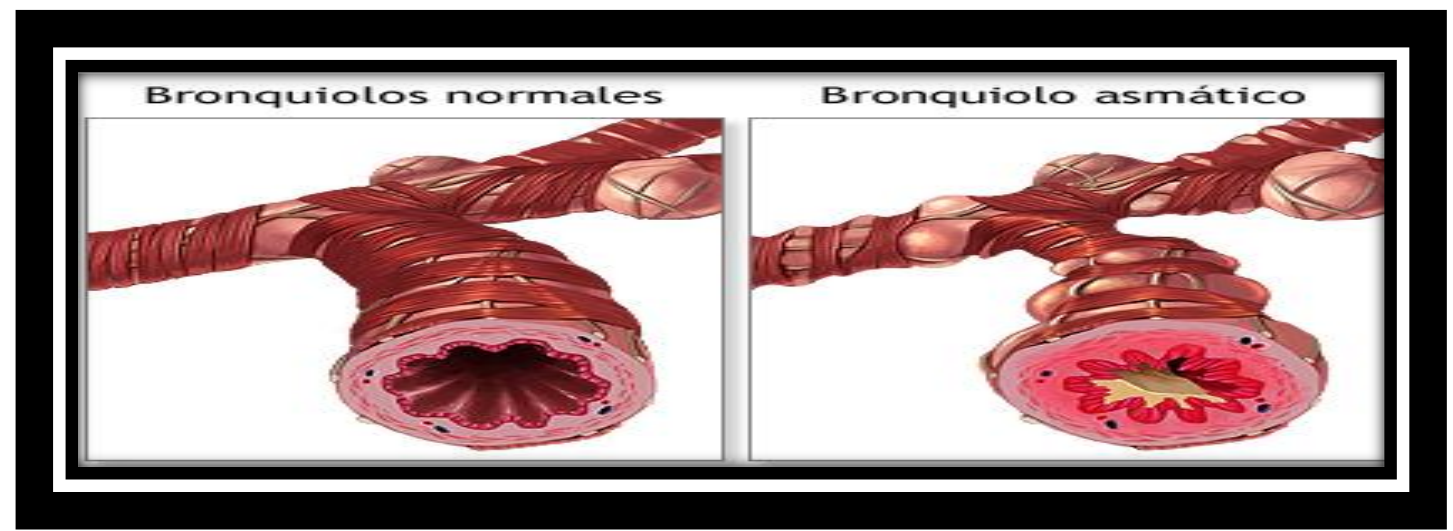

Fuente: Paz (ob.cit)

De acuerdo con esta figura, se puede indicar que, el asma es una enfermedad es causada por una inflamación (hinchazón) de las vías respiratorias. Cuando se presenta un ataque de asma, los músculos que rodean las vías respiratorias se tensionan y su revestimiento se inflama. Esto reduce la cantidad de aire que puede pasar por estas. En las personas con vías respiratorias sensibles, los síntomas de asma pueden desencadenarse por la inhalación de sustancias llamadas alérgenos o desencadenante entre los cuales se pueden citar: polen, ácaros del polvo, algunos medicamentos como ácido acetilsalicílico o aspirina u otros AINE, químicos del aire o alimentos, 


\section{Efectos de la vitamina $D$ en prevención del asma}

Vol. 3, núm. 2., (2019)

Melissa Paulina Pereira Pontón; Jheison David Cabrera Ruilova; María José Miranda Laborda; Lenin Eduardo Granda Álvarez

moho, infecciones respiratorias como refriados, estrés, humo de tabaco entre otros, que traen como consecuencia la inflamación de los bronquiolos.

De este modo, se precisa que el asma como enfermedad inflamatoria de las vías aéreas, que se caracteriza por un incremento de la respuesta de las vías aéreas respiratorias a varios estímulos y que se manifiesta por estrechamiento de las mismas, cuya severidad varía bien espontáneamente o bajo la acción del tratamiento. Su característica, por tanto, es la de presentar amplias variaciones, durante cortos períodos de tiempo, en la resistencia al flujo aéreo intrapulmonar. La reversibilidad es la característica principal del asma, que la diferencia de otras enfermedades de las vías respiratorias. A su vez, la presencia de eosinofilia en esputo, habitual en el asma, puede diferenciar a éste de otras enfermedades crónicas que se asocian con broncoespasmo reversible, como sucede con la enfermedad pulmonar obstructiva crónica.

\section{Vitamina D en la Prevención del Asma}

Los humanos obtienen la vitamina D como consecuencia de su exposición a la luz solar (principal determinante) a través de la dieta (Vitamina D3 o Colefalciferol en productos de origen animal y Vitamina D2 o Ergocalciferol de origen vegetal) y la suplementación. La vitamina D ejerce diversas funciones en todo el organismo, incluido el sistema inmunológico. Se sintetiza en la piel expuesta a la luz solar; la menor permanencia al aire libre, una característica de la vida moderna, explicaría en parte la prevalencia en aumento de insuficiencia de vitamina D.

Los estudios epidemiológicos mostraron fuertes asociaciones entre el asma y los niveles séricos reducidos de 25-hidroxitamina D (25OHD), el principal precursor en la circulación y el 


\section{Efectos de la vitamina $D$ en prevención del asma}

Vol. 3, núm. 2., (2019)

Melissa Paulina Pereira Pontón; Jheison David Cabrera Ruilova; María José Miranda Laborda; Lenin Eduardo Granda Álvarez

que mejor refleja el estado corporal de la vitamina. La vitamina D tiene efectos importantes en la modulación del sistema inmune y se cree que tenga un efecto sobre el asma es una enfermedad en la que está involucrado el sistema inmune. Hasta ahora, los datos existentes relacionados con la vitamina D y el asma se han obtenido en la población pediátrica y no han sido coherentes. Los nuevos resultados agregan más evidencia a la relación entre vitamina D y asma, sugiriendo un efecto favorable de la vitamina en la reducción de los ataques de asma. Según los científicos, aumentar los niveles de vitamina D es algo que fácilmente se puede hacer para mejorar la calidad de vida de los pacientes.

El uso de suplementos podría tener sentido para los pacientes con deficiencia de vitamina D. El asma, que produce inflamación y constricción de las vías aéreas, se ha convertido en una enfermedad común en los últimos años. Si bien la enfermedad no tiene cura, pude ser manejada con medicación y evitando alergenos y otros agentes desencadenantes.

La acción de la vitamina $\mathrm{D}$ en los casos de asma bronquial podría deberse a su papel inmunomodulador sobre las células del sistema inmunitario. Se demostró que los linfocitos T reguladores son capaces de inhibir la respuesta de tipo Th2 en el asma bronquial, mientras que la vitamina D puede estimular la acción de los linfocitos T reguladores y la secreción de interleuquina (IL10), que es una citoquina antiinflamatoria potente. La vitamina D también actúa a nivel de la subpoblación de linfocitos Th17, con disminución de la secreción de IL-17, que es una citoquina proinflamatoria asociada con la presencia de asma grave. Las concentraciones disminuidas de vitamina D provocan un desequilibrio proinflamatorio, con niveles aumentados de IL-17 proinflamatoria y disminuidos de IL-10 antiinflamatoria, con mayor gravedad del asma. 


\section{Efectos de la vitamina $D$ en prevención del asma}

Vol. 3, núm. 2., (2019)

Melissa Paulina Pereira Pontón; Jheison David Cabrera Ruilova; María José Miranda Laborda; Lenin Eduardo Granda Álvarez

Desde hace varios años se ha sugerido un papel patogénico de la vitamina D tanto en el desarrollo como en el curso del asma. Existen datos de estudios poblacionales que muestran una mayor prevalencia de déficit de vitamina $\mathrm{D}$ en niños asmáticos comparado con controles. Además, el déficit de vitamina $\mathrm{D}$ se asocia con una mayor probabilidad de presentar exacerbaciones graves en niños con asma persistente leve-moderada. Asimismo, los que presentan déficit de vitamina D tienen una reducción de la función pulmonar, mayor reactividad bronquial al ejercicio y aumento de las necesidades de corticoides inhalados. Por contra, se han asociado niveles elevados de vitamina $\mathrm{D}$ con una mejor función pulmonar, menor hiperreactividad bronquial y mejor respuesta a glucocorticoides.

Diversos estudios han analizado la relación entre exposición prenatal a la vitamina D y el desarrollo de asma en la infancia, con resultados muy dispares. En un estudio reciente se ha encontrado que en niños asmáticos con déficit de vitamina $\mathrm{D}$ y asma grave resistente al tratamiento existe un aumento del músculo liso bronquial y un peor control de la enfermedad. Infección de vías respiratorias la mayor incidencia de infecciones respiratorias durante el invierno, coincidiendo con una menor exposición solar y niveles séricos subóptimos de vitamina $\mathrm{D}$, ha servido como base para establecer una hipotética relación entre los niveles de esta vitamina y una mayor susceptibilidad a desarrollar infecciones respiratorias. Como se mencionó previamente, la vitamina D ejerce una acción moduladora.

Por lo tanto, las acciones pleiotrópicas de la vitamina D, existe cierta plausibilidad biológica de un potencial papel patogénico del déficit de esta vitamina en el desarrollo de diversas enfermedades respiratorias. Sin embargo, los numerosos estudios epidemiológicos que han 


\section{Efectos de la vitamina $D$ en prevención del asma}

Vol. 3, núm. 2., (2019)

Melissa Paulina Pereira Pontón; Jheison David Cabrera Ruilova; María José Miranda Laborda; Lenin Eduardo Granda Álvarez

encontrado asociación entre niveles bajos de vitamina D y mayor riesgo de desarrollar diversas enfermedades respiratorias o de conllevar un peor pronóstico no permiten demostrar causalidad. Uno de los principales efectos asociados a la vitamina D es su propiedad inmunomoduladora. A nivel celular, la vitamina D modifica el patrón de respuesta inmune mediante la regulación del balance de citoquinas Th1/Th2, suprimiendo las Th1 (IL-2, IL-12, INF) e incrementando la producción de Th2 (IL-5, IL-10), además mantiene la homeostasis inmune a través de la supresión Th17 (IL-17) e inducción de células T reguladoras CD4+CD25+19. Adicionalmente, células inmunes como los macrófagos activados y células dendríticas han demostrado una producción local de 1,25 (OH)2 D, la que es capaz de regular la expresión de proteínas antimicrobianas como las calecidinas y defensinas cuyo efecto restringe la respuesta inflamatoria y atenúa la severidad de los fenotipos alérgicos.

Al respecto, Martineau y Cols (2015) evaluaron la eficacia de la administración de la vitamina D en la reducción del riesgo de desarrollar exacerbaciones severas de asma y mejorar el control de los síntomas demostraron en sus resultados que: "la mayoría con asma moderada a severa, al administrar vitamina $\mathrm{D}$ redujo la tasa de exacerbaciones que requieren corticoides a razón de 71\%”.(p.54) Esto datos, llevan a resaltar, la actuación significativa que tiene para los pacientes con asma, el uso de la vitamina $\mathrm{D}$, pues, mediante ella se logra reducir las exacerbaciones y se reduce la incorporación de corticoides, que agregan al paciente otras consecuencias.

Desde un punto de vista epidemiológico, a menor número de horas de exposición solar, se constata una prevalencia mayor de asma, lo que parece tener relación con la producción de vitamina D que en su $90 \%$ se realiza en la piel a través de la interacción con la del sol. Por otra 


\section{Efectos de la vitamina $D$ en prevención del asma}

Vol. 3, núm. 2., (2019)

Melissa Paulina Pereira Pontón; Jheison David Cabrera Ruilova; María José Miranda Laborda; Lenin Eduardo Granda Álvarez

parte, los estudios clínicos realizados en niños han mostrado que niveles más bajos de vitamina D se asocian a un asma bronquial de evolución más grave, incluyen exacerbaciones frecuentes y graves, un mayor número de consultas a urgencias y hospitalizaciones.

En consecuencia, la vitamina D, de nomenclatura química 25 hidroxivitamina D3 (25 OH) D3), es una hormona esteroidea, formada a partir del metabolismo del colesterol; ampliamente conocida como hormona lipofílica, que cumple importantes funciones en la homeostasis del hueso y del calcio. Desde finales del siglo XX se comenzó a profundizar en sus funciones en el sistema inmune, a partir del conocimiento de la presencia de receptores para la vitamina D en la superficie de células como linfocitos $\mathrm{T}$ y $\mathrm{B}$, macrófagos y células dendríticas, lo que sugirió que se involucraba en la inmunoregulación del sistema inmune.

De lo antes expuesto, se puede decir que mediante la incorporación de la vitamina D los mecanismos fisiopatológicos involucrados en el asma resistente a los corticoides serían algo distintos. La colonización de las vías aéreas con bacterias proinflamatorias como Haemophilus influenzae, el estrés oxidativo (asociado con la contaminación del aire) y la deficiencia de vitamina D ejercerían un papel importante en este tipo de asma. La vitamina $\mathrm{D}$ aumenta las vías antimicrobianas e induce respuestas antioxidantes. El resultado final de las respuestas inmunológicas anormales en el asma es el remodelado de las vías aéreas, asociado con contracción del músculo liso y secreción de moco a corto plazo, y con remodelado y fibrosis en el largo plazo. La vitamina D evita la proliferación de las células de músculo liso de las vías aéreas. 


\section{Efectos de la vitamina $D$ en prevención del asma}

Vol. 3, núm. 2., (2019)

Melissa Paulina Pereira Pontón; Jheison David Cabrera Ruilova; María José Miranda Laborda; Lenin Eduardo Granda Álvarez

\section{Conclusiones.}

El análisis que caracteriza al presente artículo, lleva a precisar una serie de aportes en cuanto a los efectos de la vitamina $\mathrm{D}$ en la prevención del asma, los mismos, permiten ampliar los diferentes argumentos presentados a fin de lograr un conocimiento científico ajustado a las características del tema tratado. Entre ellas se consideran las siguientes:

A pesar de los avances en el diagnóstico y el tratamiento, el asma bronquial sigue siendo una madeja por desenredar, con múltiples fenotipos que tienen su base en su variada etiopatogenia. El asma, como ocurre con todas las enfermedades crónicas, el riesgo de desarrollar la enfermedad crece con el tiempo y la exposición a los factores de riesgo se acumula.

Otro aspecto de importancia en relación a la actuación de la vitamina D en la prevención del asma, es su actuación ante aquellos procesos de infección viral, los cuales alteran las respuestas antivirales. Es allí, donde la recomendación de la vitamina D se asocia con el aumento de las respuestas inmunológicas antimicrobianas, por medio de diversos mecanismos, entre ellos la mayor producción de péptidos antimicrobianos, como catelicidina, y la autofagia, un mecanismo importante en las infecciones virales y bacterianas.

De igual manera, se puede resaltar que, por el momento, no se conoce el esquema óptimo para indicar suplementos de vitamina D. Por ejemplo, en un metanálisis reciente de trabajos en los cuales se aportó vitamina D con el objetivo de evitar infecciones del tracto respiratorio, el tratamiento con dosis regulares se asoció con efectos protectores, mientras que los esquemas con dosis altas en bolo no indujeron protección. Es probable, también, que la corrección del estado de 


\section{Efectos de la vitamina $D$ en prevención del asma}

Vol. 3, núm. 2., (2019)

Melissa Paulina Pereira Pontón; Jheison David Cabrera Ruilova; María José Miranda Laborda; Lenin Eduardo Granda Álvarez

vitamina D mediante la mayor exposición a la luz solar sea más apropiada en el asma, en comparación con los suplementos orales. El aporte de vitamina D podría ser más útil en los niños respecto de los adultos.

Con excepción del asma resistente a los corticoides, los distintos endotipos de asma no han sido estudiados en detalle en los estudios clínicos controlados. Aunque sin duda se requieren más trabajos para responder estos interrogantes, la información en conjunto sugiere que el estado óptimo de la vitamina D es importante en términos de la aparición y la evolución clínica del asma. En esta misma dirección, la vitamina D se asocia con la inducción de tolerancia inmunológica, efectos antivirales y antibacterianos, garantiza la maduración pulmonar, de modo que se reduce el riesgo de aparición de enfermedad crónica sintomática de las vías aéreas. Durante la vida intrauterina, la vitamina D regula la expresión de ciertos genes, importantes para el desarrollo pulmonar temprano y tardío.

\section{Bibliografía.}

Alaei, E. (2019). Diferencia de Vitamina D y su Impacto en la Gravedad del Asma en Niños. Siicsalud, 15-21.

Alonso, A. (2017). Vitamina D. Presente y Futuro. Cardiología, 8-12.

Arias, F. (2012). El Proyecto de Investigación. Caracas: Episteme.

Martineau, V y Cols, A. (2015). Vitamina D. Infecciones y Asma. Médica Española, 54-62.

Paz, M. (2018). Diagnótico y Tratamiento del Asma. IMBIOMED, 32-38.

Salud, O. M. (3 de marzo de 2015). www.who.int/respiratory/about_topic/es. Obtenido de www.who.int/respiratory/about_topic/es

Soto, V. (2018). Exacerbación del Asma. Médica México, 22-29. 
Efectos de la vitamina $D$ en prevención del asma

Vol. 3, núm. 2., (2019)

Melissa Paulina Pereira Pontón; Jheison David Cabrera Ruilova; María José Miranda Laborda;

Lenin Eduardo Granda Álvarez

Valdez, J. (2019). Métodos y Procedimientos. México: Limusa.

$$
\text { (9)(1)(Q) }
$$

RECONOCIMIENTO-NOCOMERCIAL-COMPARTIRIGUAL

CC BY-NC-SA

ESTA LICENCIA PERMITE A OTROS ENTREMEZCLAR, AJUSTAR Y CONSTRUIR A PARTIR DE SU OBRA CON FINES NO

COMERCIALES, SIEMPRE Y CUANDO LE RECONOZCAN LA AUTORÍA Y SUS NUEVAS CREACIONES ESTÉN BAJO UNA LICENCIA CON LOS MISMOS TÉRMINOS. 